\title{
The d-AICuRh stability - the proof of the random-tiling hypothesis by the distribution moments analysis
}

\author{
I. Buganski, R. Strzalka, J. Wolny \\ AGH University of Science and Technology, Faculty of Physics and Applied Computer Science, Krakow, Poland \\ ireneusz.buganski@fis.agh.edu.pl
}

The atomic structure of the decagonal $\mathrm{Al}-\mathrm{Cu}-\mathrm{Rh}$ quasicrystal with a space group is refined based on five X-ray diffraction datasets, collected at $293 \mathrm{~K}, 1013 \mathrm{~K}, 1083 \mathrm{~K}, 1153 \mathrm{~K}$ and $1223 \mathrm{~K}$ with the use of a synchrotron radiation [1]. The real-space structure solution with the tiling-and-decoration approach based on the moment series expansion [2] is executed.

All the crystallographic $R$ - factors are ranging from $5.9 \%$ to $6.4 \%$ for the datasets of common 1460 symmetry-inequivalent peaks. What is the most intriguing is the correlation (Pearson correlation equal to 0.85 ) between lattice parameters (edge-length of rhombus and the interatomic layer distance) and the maximum of the residual electron density. The identical temperature dependence presented in figure 1 for the parameters implies the phase transformation. The residual density is agglomerated in the origin of the 4D unit cell what implies the phase transformation is related to the General Penrose Tiling (GPT). Additionally, we can observe a local minimum around the $1083-1153 \mathrm{~K}$ of the moments values being directly related to phasons. This is the temperature the structure is the most stable around. The existence of the local minimum in all the plots proves the phason disorder is related to the structure stability what was previously questioned due to insufficient quality of the refinement [1].

We modified the moment series approach to accommodate the existence of the $5^{\text {th }}$ atomic surface arising for the random-tiling model of the decagonal quasicrystal (figure 2). After the structural refinement with the updated model we obtained much better results in terms of the R-factors. Even more, up to the uncertainty estimated with Hesse matrix, we could prove the $5^{\text {th }}$ atomic surface existence is not only the artefact of the electron density calculation but the crucial feature of the structure in the 1083-1153 K. The calculations prove the random-tiling hypothesis of the structural stability is true for the decagonal quasicrystals and the structure is stabilized by phasons.

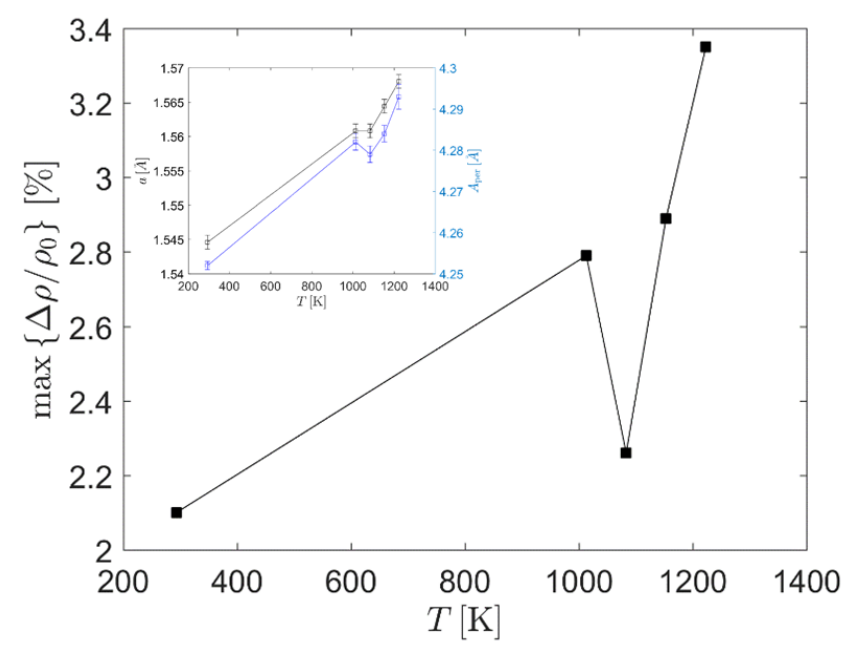

Figure 1. The temperature dependence of the lattice parameter (inset) and the maximum of the residual electron density. The local minimum in the point of the structure stability is visible.

[1] Kuczera, P., Wolny, J., Steurer, W. (2014). Acta Cryst. B70, 306-314

[2] Buganski, I., Strzalka, R., Wolny, J. (2020). J. Appl. Cryst. 53, 904-913

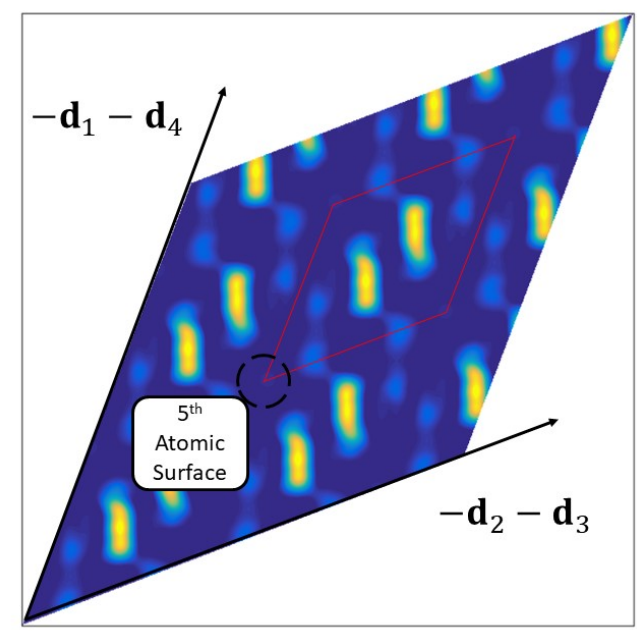

Figure 2. The 2D section through the $4 \mathrm{D}$ electron density (spanned by $\mathbf{d}$ vectors of the decagonal basis). The residual density in the position of the $5^{\text {th }}$ atomic surface of the GPT is encompassed with dotted-line circle.

Keywords: quasicrystal; phason; structure stability 